\title{
Progress in Understanding of Hairy Cell Leukemia and Hairy Cell Leukemia-Like Disorders
}

\author{
Nahla AM Hamed* \\ Faculty of Medicine, Alexandria University, Egypt \\ Submission: October 18, 2018; Published: November 05, 2018 \\ "Correspondence Address: Nahla AM Hamed, Professor of Hematology, Faculty of Medicine, Alexandria University, Egypt
}

\begin{abstract}
HCL is a well-recognized clinicopathological entity. BRAF-V600E mutation is present in many patients with cHCL and is absent in vHCL. Despite the remarkable progress that has been made with purine nucleoside analogs as the initial treatment of HCL patients, yet relapse and eventual development of refractory disease occurs. An appropriate approach to retreatment represents continuing areas for research. FDA has recently approved immunotoxin conjugates moxetumomab pasudotoxfor the treatment of HCL patients who have already undergone at least two lines of standard therapy. The development of internationally accepted, reproducible criteria on definitions of response, relapse, and methods of determining MRD is of paramount importance in comparing clinical trials.
\end{abstract}

Abbreviation: HCL: Hairy Cell Leukemia; MRD: Minimal Residual Disease; vHCL: Hairy Cell Leukemia Variant; cHCL: Classic HCL; IHC: Immunohistochemical

\section{Introduction}

HCL is a rare subtype of B-cell chronic lymphoid leukemia[1] that accounts for $2 \%$ of all leukemia [2].Males are affected more frequently than females (ratio 4:1), usually over the age of 60 years [3]. It is characterized by progressive pancytopenia, splenomegaly [1] (approximately 90\%) [4] and infiltrations of the bone marrow, liver, and spleen, with B lymphocytes possessing cytoplasmic projections that appear as fine hair-like microvilli. [1] Some patients present with profound pancytopenia accompanied by massive splenomegaly. Others have moderate asymptomatic pancytopenia that may remain progression-free for many years without therapy [4]. Monocytopenia is a relatively sensitive and specific manifestation of cHCL[4]. There are numerous case reports of atypical manifestations of HCL. For example, HCL may atypically present with cutaneous, visceral, bone, pleural, and meningeal involvement [5].

HCL can mimic or coexist with other clonal hematologic disorders and has been associated with autoimmune disorders [5].It may coexist with other B-lymphoid malignancies. Polyclonal and monoclonal gammopathy have been reported in 3\%-20\% of patients and may be related to an associated plasma cell dyscrasia, lymphoma, or autoimmune disorder. HCL may be associated with a variety of autoimmune disorders, most commonly vasculitis [5]. HCL must be differentiated from other HCL-like disorders, including vHCL and splenic diffuse red pulp lymphoma[2].

\section{Hairy Cell Leukemia Variant (vHCL)}

vHCL should be suspected in cases presented with splenomegaly associated with cytopenias, a normal monocyte count, and lymphocytosis characterized by hairy cells with prominent nucleoli. Despite morphologic similarities, vHCL is not biologically related to CHCL[6]. CHCL and vHCL differ in prognosis, with vHCL responding poorly to PAs, with a median survival less than half that of cHCL[7].

\section{VH4-34 Positive HCL}

A subset and a new variant of HCL that is associated with poor prognosis, which includes higher disease burden at diagnosis, poor response to standard therapy, shorter overall survival and absence of the BRAF-V600E mutation [2].Forty percent of vHCL and ten percent of HCL patients have an IGHV4-34 immunoglobulin variable heavy chain rearrangement [2].

\section{The Genetic Bases of cHCL and vHCL}

Gene-expression profile data strongly suggest that hairy cells arise or are related to memory B cells [5].The mutations present in each HCL subtype are distinct [8]. BRAF-V600E mutations are identified in up to $80-90 \%$ of HCL cases [2]. The mutation in B-raf proto-oncogene (BRAF gene) (7q34) occurs in exon 15 at position 1799 , in which thymine and adenine are exchanged, leading to valine (V) substitution by glutamate (E) at codon 600 (V600E) of 
the BRAF protein. BRAF-V600E mutation constitutively activates BRAF by autophosphorylation of the protein and downstream MEK-ERK signaling pathway, leading to increased expression of genes involved in survival and proliferation [2]. In BRAF-V600Enegative HCL cases, the possibility of 2 novel mutation in exon 11 (F468C, D449E) must be excluded. (1)BRAF-V600E mutation is not detected in vHCL [8], IGHV4-34 variants of HCL [1] or other small B-cell lymphoid neoplasms [8].

The most common genetic alteration in cHCL apart from BRAFV600E was heterozygous loss of chromosome7q, the minimally deleted region of which targeted wild-type BRAF, subdividing cHCL into those hemizygous versus heterozygous for the BRAFV600E mutation [7]. Additional alterations are frequently required for tumor initiation and/ or progression as suggested by studies of BRAF-V600E-mutant cells in diverse cancers marked by BRAF-V600E mutation [7]. The two mutations most frequently identified after BRAF mutations were histone methyltransferase KMT2C (MLL3) and CDKN1B mutations, which occur in 15\% and $11 \%$ of patients, respectively [2].

The CDKN1B gene encodes the cyclin dependent kinase inhibitory protein p27, which inactivates the cyclin E-CDK2 complex. The p27 protein is a known tumor suppressor that exerts either stimulatory or inhibitory effects on cell proliferation, cell motility, or apoptosis. Moreover, p27 upregulation can participate in chemotherapy resistance. At high levels, p27 binds to the cyclin E-CDK2 complex, inhibiting its activity and allowing cell cycle arrest. In contrast, lower levels of p27 protein stabilize cyclin D-CDK4/6 complexes and facilitate cell cycle progression [1].

Mutation in MAP2K1which encodes MEK1 (downstream of BRAF) have been reported in almost half of vHCL and in the majority of HCL that use IGHV4-34 [8]. Activating mutations in CCND3 and a change-of-function mutation in the splicing factor U2AF1 were present in $13 \%$ for any in vHCLs[7]. Recurrent inactivating mutations in KMT2C (MLL3) were identified in 25\% of vHCLs[7]. These data identify numerous novel drivers of HCL and additional shared cooperating alterations, as well as diseasespecific alterations targeting BRAF, KMT2C, and CDKN1B in CHCL and MAP2K1, CCND3, U2AF1, TP53, and KMT2C in vHCL [7].

\section{Diagnosis}

Diagnosis of HCL is based on morphological evidence of hairy cells, an HCL immunologic score based on the four markers CD11C, CD103, CD123, and CD25 expression. A score of 3 or 4 is observed in $98 \%$ of HCL cases, whereas the score in other HCL-like disorders is usually 0 or 1 [2]. cHCL and vHCL share expression of CD11c and CD103, only cHCL expresses CD25, CD123, CD200, and annexin A1 [7].The immunophenotype CD103pos/CD11cbright/ CD123neg/CD25neg supports the diagnosis of vHCL [6].

A trephine bone marrow biopsy and aspirate are important at diagnosis. A dry bone marrow tap is often attainable because of extensive fibrosis. Approximately $10 \%$ of patients will have a hypocellular bone marrow biopsy at diagnosis reflecting a decrease in normal hematopoiesis [4]. IHC stains for CD20, CD76, annexin A1, and VE1 (a BRAF-V600E stain) will establish the diagnosis and provide an accurate assessment of the degree of leukemic bone marrow infiltration. In patients treated with antiCD20 monoclonal antibody apply pan B-cell markers, other than CD20, such as CD79a and/or HCL-specific markers (e.g., VE1) or DBA.44 to avoid unpredictable results.

Bone marrow biopsy should be delayed 4 to 6 months after cladribine administration and performed after a clinical response with pentostatin therapy to document a complete remission[2]. Highly sensitive techniques (e.g., allele-specific polymerase chain reaction or next-generation sequencing) should be preferred to avoid false-negative results when few leukemic cells $(<10 \%)$ are present in the peripheral blood or in diluted bone marrow aspirates. If leukemic cells are insufficient or highly sensitive molecular techniques for genomic profiling are inaccessible, IHC stain (eg, VE1) to the bone marrow biopsy may enable detection of BRAF-V600E mutation [4].Demonstration of BRAF-V600E mutation could be important for those who do not respond to standard therapy or have multiple relapses [4].Flow cytometry, using an 8-color panel (CD103/CD305/CD19/CD123/CD25/ CD3/CD45/CD20), can be a tool for detecting blood MRD [2].

\section{Treatment}

\section{When to Start Treatment}

Treatment should be started in symptomatic patients (e.g., symptomatic splenomegaly) or if the hematologic parameters are declining. At least one of the following hematologic parameters indicates a need for treatment: hemoglobin $<11 \mathrm{~g} / \mathrm{dL}$, platelet count $<100.000 / \mathrm{mL}$ or absolute neutrophil count $<1000 / \mathrm{mL}$ [4].

\section{Purine Nucleoside Analog (PAs)}

Primary induction therapy for HCL involves either cladribine or pentostatin [4]. They are associated with profound and prolonged myelosuppression and immunosuppression[9]. Attempts to control infection should be pursued prior to starting the PAs [2]. Resistance and relapses occur with PAs[5]. Splenomegaly $(>3 \mathrm{~cm})$, leukocytosis $\left(>10 \mathrm{X} 10^{9} / \mathrm{L}\right)$, hairy cells in the blood ( $>5 \times 10^{9} / \mathrm{L}$ ), and high beta2-microglobulin (> $2 \mathrm{~N}$ ) are associated with a poor prognosis and resistance to PAs. CD38 expression drives poor prognosis.Patients with unmutated IGHV have shorter overall survival durations than those with the mutated gene [2]. Resistance is a major problem with vHCL[5]. Activation of signaling pathways parallel to the MAPK pathway may be responsible for RAF inhibitor resistance [7]. Relapses in HCL are in the range of $20 \%-30 \%$ at 5 years and up to $48 \%$ at 10 years [5].

\section{Alpha-Interferon (IFN)}

Alpha-IFN can be an alternative in pregnant women and can be used transiently if infection control is not possible prior to PAs institution [2]. 


\section{Assessment or Response}

Complete Response: It is defined by normalization of peripheral blood counts (without transfusion), resolution of palpable splenomegaly and disappearance of hairy cells from the bone marrow [2].Minimal residual disease (MRD) is defined as HCL infiltrates recognizable by IHC stains, but not by conventional stains [4]. A single report grouped the risk of relapse predicted by MRD into: group I, $<1 \%$ positive cells, low risk for relapse; group II, $1 \%$ to $5 \%$ positive cells by IHC, intermediate risk for relapse; and group III, $>5 \%$ positive cells by IHC, higher risk for relapse. Future studies are needed to validate the clinical value of these predictive groups [4].

Partial Response is defined by near normalization of the peripheral blood counts with a minimum of $50 \%$ improvement in both organomegaly and bone marrow biopsy infiltration with hairy cells [4].

\section{First Relapse}

Relapse retreatment is based upon recurrence of diseaserelated symptoms or deterioration in hematologic parameters equivalent to initial values used for beginning treatment [4]. Therapeutic options will depend on the duration of first remission [2]. Relapse after over 60 months remission should be re-treated with the same or an alternative PAs. For remissions $>24$ months, use PAs followed by rituximab. Response to either humanized, glycoengineered Type II (obinutuzumab) or the second-generation anti-CD20 monoclonal antibodies (ofatumumab) is not yet known. Patients with relapse before 24 months should be considered as relapsed/refractory HCL patients. The presence of a BRAF-V600E mutation must be checked. Specific BRAF inhibitors represent the best option for mutated BRAF cases.In unmutated BRAF cases, immunotoxins, BCR inhibitors, combination of bendamustine with rituximab or investigational agents and regimens should be considered.

\section{Novel Agents}

a. FDA has recently approved immunotoxin conjugates moxetumomab pasudotox (lumoxiti), a bacterial toxin-based drug for the treatment of HCL patients who have undergone at least two lines of standard therapy. It binds CD22 receptors on the surface of cancerous B cells where it is internalized and processed to release its toxic payload. However, there is a risk of capillary leak syndrome [10].

b. Ibrutinib, a Bruton tyrosine kinase inhibitor that is currently under study for HCL patients who have not achieved optimal response to standard therapies [4].

c. BRAF inhibitor (Vemurafenib, dabrafenib): Vemurafenib is an ATP-competitive BRAF inhibitor that has potent antitumor activity in BRAF-V600E-mutated HCL patients.
Its administration has improved peripheral blood counts. Vemurafenib has been used successfully in a neutropenic patient with an active infection. After the associated infection of the underlying leukemia has been resolved, consolidation with standard therapy could be approached. Genomic analysis of de novo vemurafenib-resistant cHCL identified a novel gain-of-function mutation in IRS1 and losses of NF1 and NF2. Adverse effects from the BRAF inhibitors may include skin rash, arthralgias, arthritis, or secondary skin tumors [4]. Rarely, vemurafenib has caused abnormal renal function and some fewer common toxicities.

d. Consider investigational approaches if poor-risk features were identified (e.g., severe anemia, spleen $>10 \mathrm{~cm}$ below the left costal margin, atypical immunophenotypic profile, p53 mutation, IGHV4-34 rearrangement, unmutated IGHV, absence of BRAF-V600E mutation, identification of an underlying explanation for a less-than desired initial response.

\section{Conclusion}

Despite the substantial progress in managing HCL leukemia, the disease has not been cured and requires continued organized clinical investigation to explore novel agents and strategic combination.

\section{References}

1. Robak T, Smolewski P (2015) New mutation in hairy cell leukemia. Blood 2015;126(8):930-932.

2. Troussard X, Cornet E (2017) Hairy cell leukemia 2018: Update on diagnosis, risk-stratification, and treatment. Am J Hematol 92: 13821390.

3. Montserrat E, Hillmen P (2016) Chronic lymphocytic leukemia and other chronic B-cell disorders. In: Hoffbrand AV, Higgs DR, Keeling DM, Mehta AB (Eds), Postgraduate hematology. ( $7^{\text {th }}$ edn), Wiley-Blackwell, Oxford, UK.

4. Grever MR, Abdel-Wahab O, Andritsos LA, Banerji V, Barrientos J, et al. (2017) Consensus guidelines for the diagnosis and management of patients with classic hairy cell leukemia. Blood 129(5): 553-560.

5. Wanko SO, de Castro C (2006) Hairy Cell Leukemia: An elusive but treatable disease. The Oncologist 11: 780-789.

6. Ferreira TZ, Sandes AF (2016) Lymphocytosis, villi, and nucleoli: a variant of hairy cell leukemia. Blood 128: 1018.

7. Durham BH, Getta B, Dietrich S, Taylor J, Won H, et al. (2017) Genomic analysis of hairy cell leukemia identifies novel recurrent genetic alterations. Blood 130(14): 1644-1648.

8. Swerdlow SH, Campo E, Pileri SA, Harris NL, Stein H, et al. (2016) The 2016 revision of the World Health Organization classification of lymphoid neoplasms. Blood 127(20): 2375-2390.

9. Grever MR (2016) BRAF inhibitor: targeted therapy in hairy cell leukemia. Blood 127(23): 2784-2785.

10. NCI Staff (2018) Moxetumomab approved by FDA for hairy cell leukemia. 


\section{Your next submission with Juniper Publishers} will reach you the below assets

- Quality Editorial service

- Swift Peer Review

- Reprints availability

- E-prints Service

- Manuscript Podcast for convenient understanding

- Global attainment for your research

- Manuscript accessibility in different formats ( Pdf, E-pub, Full Text, Audio)

- Unceasing customer service

Track the below URL for one-step submission https://juniperpublishers.com/online-submission.php 